\title{
Género y trabajo: una intersección en construcción. Análisis de dos empresas recuperadas
}

\author{
María de los Ángeles Dicapua \\ Universidad Nacional de Rosario (UNR), Argentina \\ Melina Perbellini \\ Universidad Nacional de Rosario (UNR), Argentina
}

Evangelina Tifni

Universidad Nacional de Rosario (UNR), Argentina

Género y trabajo: una intersección en construcción. Análisis de dos empresas recuperadas

Resumen: El presente artículo presenta las conclusiones de una investigación realizada en dos empresas recuperadas del área del Gran Rosario (Provincia de Santa Fe - Argentina). El objetivo fue comparar el proceso de lucha para recuperar las fuentes de trabajo que llevaron adelante un grupo de mujeres trabajadoras o esposas de trabajadores de cada empresa estudiada, constituyendo nuevos modelos de funcionamiento técnico y organizacional. La hipótesis de trabajo fue que para cada caso estudiado predominó una "lógica particular de funcionamiento" que derivó en procesos de cambio en las identidades laborales y de género de los actores. La metodología utilizada fue de carácter cualitativo, a partir de la triangulación de datos en el proceso de análisis. Se efectuaron entrevistas en profundidad y se realizaron observaciones no participantes dentro de las fábricas.

Palabras claves: género, trabajo, empresas recuperadas.

Gênero e trabalho: uma interseção em construção. Análise de duas empresas recuperadas

Resumo: O presente artigo apresenta as conclusões de uma pesquisa em duas empresas recuperadas da região do Gran Rosario (Provincia de Santa Fé - Argentina). O objetivo foi comparar o processo de luta para recuperar as fontes de trabalho de um grupo de mulheres trabalhadoras ou de esposas de trabalhadores em cada empresa estudada, conformando novos modelos de funcionamento" técnico e organizacional. A hipótese de trabalho para cada caso estudado foi a predominância de uma "lógica particular de funcionamento, que derivou em processos de mudança nas identidades laborais e de gênero dos atores. A metodologia foi de caráter qualitativo, a partir da triangulação de dados no processo de análise. Foram efetuadas entrevistas em profundidade e foram realizadas observações não participantes nas fábricas.

Palavras-chave: gênero, trabalho, empresas recuperadas.

\section{Gender and Work: an Intersection in Construction. Analysis of Revived Companies}

Abstract: This article presents the conclusions of a study conducted at two companies recovered through the formation of labor cooperatives in the Greater Rosario region in Santa Fé Province, Argentina. The objective was to compare the process of struggle to revive the sources of work of a group of working women or for the wives of the workers at each company studied, conforming to new models of technical and organizational operation (division of labor, forms of communication, management style, decision making processes etc). The hypotheses of labor for each case studied was the predominance of a "particular logic of operation", which derived from processes of change in the labor identities and gender of the actors involved. The research methodology was qualitative, based on the triangulation of data in the analysis process. In-depth interviews were conducted with women workers at the companies studied as well as non-participative observations at the factories.

Key words: gender, labor, recovered companies. 


\section{Introducción}

En el presente artículo serán analizadas dos “empresas recuperadas", empresas/fábricas que los trabajadores deciden ocupar con el fin de hacerse cargo de la producción y resguardar sus puestos de trabajo, cuando sus dueños las declaran en quiebra, o cuando se produce un vaciamiento patrimonial. La estrategia de ocupación y recuperación de fábricas representa una estrategia defensiva que se encuadra en la supervivencia de la empresa y en la conservación del trabajo (FAJN, 2004). Sin embargo, nace una nueva modalidad de acción cuando los trabajadores asumen un rol activo en la empresa, y totalmente diferente al que han tenido hasta ese momento. Los mismos se dan nuevas formas organizativas, donde las relaciones tradicionalmente establecidas se derogan a favor de nuevas reglas.

Las empresas estudiadas pertenecen a ramas distintas de la producción. Por un lado, una empresa de la rama metalmecánica, y por otro, una empresa de proceso. En ambas los trabajadores han atravesado largos procesos de recuperación de sus fuentes de trabajo con estrategias de lucha disímiles y donde el peso relativo de los distintos actores en juego siguió el vaivén de esas estrategias. La primera empresa analizada, persistió en su producción original como una de las cristalerías artesanales más importantes del país. En cambio la actividad original de la segunda empresa (producción de unidades carrozadas) debió adaptarse para pasar a dedicarse sólo a reparaciones menores.

La hipótesis que guió este trabajo fue que para cada caso estudiado predominó una "lógica particular de funcionamiento" (industrial en oposición a la artesanal o doméstica) (EYMARD-DUVERNAY, 1994) que derivó en procesos de cambio en las identidades laborales y de género de los actores involucrados en el proceso.

\section{Metodología}

El objetivo del presente artículo fue comparar el proceso de lucha para recuperar las fuentes de trabajo que llevaron adelante un grupo de mujeres trabajadoras o esposas de trabajadores de cada empresa estudiada, constituyendo nuevos modelos de funcionamiento técnico y organizacional (división del trabajo, formas de comunicación, estilo de gestión, procesos de toma de decisiones, etc.).

Se seleccionaron dos unidades de análisis constituidas por dos empresas recuperadas del área del Gran Rosario (Provincia de Santa Fe - Argentina), que pertenecen a ramas distintas de la producción. En cada unidad se trabajó con las mujeres involucradas en estos procesos. Para analizar las experiencias de estas mujeres se utilizó la técnica de triangulación de distintas líneas teóricas sobre el plano empírico.

La investigación se inscribió en el estudio de casos con un abordaje de tipo cualitativo. Se dio prioridad a la técnica de la entrevista estructurada y semi-estructurada y a la entrevista abierta en profundidad. Por otro lado, se trabajó con métodos biográficos o trayectorias de vida (DUBAR; DEMAZIERE, 1997) con el objetivo de recabar las experiencias subjetivas de un grupo de mujeres involucradas en la recuperación ${ }^{1}$. Por último se efectuaron observaciones no participantes en los casos testigo seleccionados con el fin de indagar en el proceso de construcción de nuevas identidades (laborales y de género).

\section{Marco teórico: identidad laboral y de género}

La identidad es una conquista que se capitaliza en el orden de lo singular pero se opera en el orden de la intersubjetividad. La realización de sí mismo pasa por la mirada del otro. En el ámbito social, la búsqueda de la identidad debe reconducirse permanentemente por medio del reconocimiento del hacer; donde me vuelvo lo que soy. Sólo accedo a mí mismo y al otro por la mediación de un hacer "considerado", tomado en cuenta. Función del espacio abierto a la dinámica del reconocimiento, el trabajo puede contribuir a la realización de sí mismo. Trabajar es entonces beneficiarse con un lugar para hacer y hacerse con los otros (DESSORS; MOLINIER, 1998).

Para Dubar (2001, p. 9),

Las identidades en el trabajo no derivan mecánicamente de las 'situaciones' definidas desde el exterior a partir de criterios 'objetivos'. [...] Estas identidades dependen sobre todo de las relaciones que se mantienen con los otros actores (jefes, colegas, etc.) de este 'trabajo vivenciado'; es decir, de una situación 'construida y definida subjetivamente'. [...] De este modo, las identidades en el trabajo se convierten en maneras de ser 'actor de sí'.

Así, la identidad laboral surgida en el acto mismo de trabajar con otros es constitutiva del sujeto, como lo es su identidad de género.

Para Arfuch (2005, p. 24), la identidad de género sería "una construcción nunca acabada, abierta a la temporalidad, la contingencia, una posicionalidad relacional, sólo temporariamente fijada en el juego de las diferencias."

Desde la perspectiva de Scott (1999), la identidad de género se compone de tres aspectos interrelacionados: en primer lugar, un conjunto de símbolos culturalmente significativos que se encuentran disponibles y que enuncian representaciones de lo femenino 
y lo masculino en contextos particulares. En segundo lugar, un conjunto de normas que se genera a partir de aquel conjunto de símbolos y de sus interpretaciones (religiosas, educativas, legales, científicas y políticas). Y por último, las prácticas cotidianas sostenidas sobre aquellas representaciones y limitadas y habilitadas por el orden normativo.

La identidad laboral y de género se reconstituye continuamente a partir de las transformaciones del contenido y la naturaleza de la vida cotidiana, especialmente con la reorganización del tiempo y el espacio. Las tendencias de estos cambios derivan en la expansión de los mecanismos de desmembración de las relaciones sociales, redefiniendo el contenido de estas identidades. En un contexto de una gran diversidad de opciones y posibilidades, el sí mismo, siguiendo a Giddens (1997), tiende reflexivamente a reconstituirse frente a sistemas expertos que, como influencias desmembradoras, tratan inútilmente de racionalizar las nuevas incertidumbres. Para este autor, estos sistemas sólo proveen criterios sujetos a permanente revisión.

La autoidentidad (GIDDENS, 1997) es el yo entendido reflexivamente por la persona en función de su biografía. La identidad del yo es esa continuidad interpretada reflejamente por el agente. Ser una "persona" no es simplemente ser un actor reflejo sino tener un concepto de persona en su aplicación al yo y a los otros. La identidad de una persona no se ha de encontrar en el comportamiento ni en las reacciones de los demás, sino en la capacidad para llevar adelante una crónica particular. Se estructura continuamente sobre la consideración del riesgo en las actividades diarias. Tanto la pluralización de los contextos de acción y de las autoridades reconocidas como la apertura de la vida social, cada vez más extendida, se explicitan en la elección del estilo de vida $^{2}$ y en la organización reflexiva del proceso de constitución de la autoidentidad.

Todos los sujetos sociales poseen una construcción biográfica previa a su construcción como tales. Esto conlleva un proceso de formación de sujetos institucionalizados, o sea, asalariados, escolarizados, ciudadano. Como bien señala Butler (2004, p. 20),

[...] la hegemonía pone el énfasis en las maneras en que opera el poder para formar nuestra comprensión cotidiana de las relaciones sociales y para orquestar las maneras en que consentimos (y reproducimos) esas relaciones tácitas y disimuladas en el poder.

Se puede afirmar que los campos hegemónicos no son espacios compactos, dentro de ellos existen luchas de sentido, disputas de intereses. Como bien plantea Laclau (2004), para que exista la hegemonía se necesita que los objetivos sectoriales de un grupo operen en nombre de una universalidad que los trascienda. Es decir, la representación es constitutiva de la relación hegemónica. Esta relación, como dice Laclau (2004, p. 62),

[...] requiere de la producción de significantes tendencialmente vacíos que, mientras mantienen la inconmensurabilidad entre universal y particulares, permite que los últimos tomen la representación del primero.

Por lo tanto, cuando se piensa en lo hegemónico nos referimos a una lucha por la imposición del significado del significante vacío. Pensar en el sector hegemónico remite directamente a la resistencia a dicho grupo. Esta resistencia se construye de maneras diferentes en los diversos ámbitos de la sociedad. Un ejemplo de esta resistencia son las diferentes estrategias de lucha que se dan los trabajadores en los procesos de toma o recuperación de sus fuentes laborales.

El quiebre de la sociedad salarial (CASTEL, 1997) llevó a revalorizar, como una preocupación central para los estudios de género, la transformación de las relaciones sociales, fundamentalmente en términos socioeconómicos. Desde esta perspectiva, se comienzan a trabajar los fenómenos de "feminización de la pobreza". Se intenta la construcción de articulaciones nuevas del concepto de género con los de grupos sociales laboralmente precarizados que, manteniendo la significación primaria de género - la asimilación de género femenino a género oprimido, invisibilizado, y la asimilación de relaciones de género como relaciones de poder-, permitan un análisis que tome en cuenta los contextos multiculturales permanentemente cambiantes (HIRATA; KEORGOAT, 1995)

Para abordar estas problemáticas se trabajó con los discursos de las mujeres que protagonizaron estos procesos de cambio, particularmente en relación a las transformaciones en sus subjetividades, que las llevaron a reconfigurar su lugar en las relaciones de trabajo y de género.

\section{Estudios de Casos}

\section{Caso 1}

El primer estudio de caso a abordar es una cristalería artesanal recuperada por sus trabajadores en el año 2002, y conformada en cooperativa de trabajo un año después. El proceso de recuperación de esta empresa tiene una particularidad: desde el momento de su cierre en 1995 hasta la vuelta a ésta en el año 2003, existen ocho años de distancia. Luego de este tiempo, 40 trabajadores vuelven a juntarse con el fin último de poner a producir la fábrica. 
La cooperativa estudiada está conformada por 70 socios-trabajadores, los cuales están divididos en cuatro secciones: horno, producción, afiladura y depósito, y administración. Cuenta con seis oficiales de banco, los cuales tienen todos más de 60 años.

Es necesario tener en cuenta que de estos 70 trabajadores, 1/3 son mujeres. La mayoría trabajan en la "sección afiladura", en la cual se encargan del tallado, pulido y revisación. En esta sección se encuentra una gran mayoría de "viejas trabajadoras" - trabajadoras de la empresa anterior. La encargada de la sección que denominaremos G. - es la misma de la empresa anterior, quien tuvo un papel fundamental en el proceso de recuperación de la empresa, y es sumamente reconocida por sus compañeras de sección. El resto de las trabajadoras se ubican en la "sección producción" - donde se encuentran todos los oficiales de banco y es el lugar de fabricación a mano y soplado del producto - y en la de "administración".

En la sección producción son todas "nuevas trabajadoras" que ingresaron directamente en la cooperativa luego de haber pasado por la "Escuela de Artesanos Cristaleros y Vidrieros" organizada por los trabajadores.

Es necesario remarcar, para comprender el proceso de recuperación y la "lógica particular de funcionamiento" - en este caso "artesanal" o "doméstica" (EYMARD-DUVERNAY, 1994) -, cómo se conforma el proceso de trabajo en una cristalería artesanal: "La organización del trabajo está basada en el predominio de la jerarquía del oficio, de cuya centralidad deviene el funcionamiento del proceso productivo" (ANGÉLICO; BUNEL, 1989, p. 18).

El "oficial de banco" es quien gestiona el trabajo. Esta situación se deriva de la capacidad de decisión autónoma de los operarios (TOURAINE, 1971).

El oficio de artesano cristalero es altamente calificado. Necesita un mínimo de diez años de aprendizaje. Esto conduce a que las categorías dentro de la cristalería estén jerarquizadas: oficial de banco, medio oficial y aprendiz. Si bien se lo puede ver como una escala ascendente hasta llegar al cargo más importe no es tan así: se puede aprender el oficio, pero, como nos dicen los trabajadores: "Para llegar a ser artesano del cristal se necesita no sólo la voluntad de aprender, sino algo más profundo: tener el 'don de artesano'."

La producción artesanal del cristal tiene dos características fundamentales: por un lado, la especialización en el oficio, que conduce al reconocimiento del hacer; donde me vuelvo lo que soy y accedo a mí mismo y al otro por la mediación de un hacer "considerado" (DESSORS; MOLINIER, 1998). Por otro lado, el trabajo en conjunto. Hay que tener en cuenta que el trabajo se organiza por equipo o "plazas, compuestas por aprendices, ayudantes y que regidas por el oficial realizan en 'serie o heterogénea' los diversos artículos" (ANGÉLICO; BUNEL, 1989, p.19).

Para la producción artesanal de, por ejemplo, una copa de cristal se necesita contar con un mínimo de 10 personas, entre foguistas, sacadores, sopladores, cuellistas, etc. A su vez, para poner en funcionamiento una cristalería se necesita como mínimo tres o cuatro plazas de personas. Esto hace que sea un trabajo en equipo, en el cual todas las piezas son imprescindibles. Como nos dicen Dessors y Molinier (1998), las relaciones de confianza y de cooperación se hacen posibles por medio de la elaboración de reglas comunes. Pero las reglas del oficio no determinan únicamente las maneras de hacer, dan paso a la construcción de, por un lado, una forma de identidad laboral, y por el otro (re)constituyen las relaciones de género dando paso a la construcción de una forma particular de identidad de género. Un ejemplo de esto lo podemos observar en los conflictos sucedidos en el primer año de funcionamiento de la Escuela de Artesanos donde la mayoría de los alumnos fueron mujeres de entre 20 y 40 años:

[...] mandaron buena plata para la Escuela, pero me trajeron todas mujeres. Yo no desmerezco a las mujeres, porque acá vos ves, hay 4 o 5 mujeres en el grupo, para 40 personas, 4 o 5 está bien, más no. Yo no quiero mandar las mujeres al horno. Por un montón de motivos, por los pechos, por un montón de cosas. Pero si yo no tengo los pibes, que son los que van para arriba, que empiezan a sacar vidrio, a hacer un colágeno, a soplar, en dos años no tenemos más nada. Cuando yo te hablo de juventud, te hablo entre 14 y 18 años. Los de 19, 20 años, ya no te sirven (Entrevista: Trabajador de la cristalería).

En el discurso anterior es notable como aparecen marcadas las "reglas del oficio". Un oficio considerado "de varones" tanto por mujeres como por hombres. Descripto como un trabajo forzoso, cansador. Los trabajadores consideran que el lugar de las mujeres dentro de la fábrica se encuentra en la "sección afiladura" y en la "sección administración". La distancia varón-mujer es muy notable en la sección producción:

Yo trabajo al igual que cualquier chico de acá. Pero la diferencia está entre el hombre y la mujer, obviamente. En el trato, un poco en la comodidad, un poco en que hago el mismo trabajo pero a los hombres no les cae bien. Siempre tenemos disputas en eso (Entrevista: Trabajadora de la Cristalería).

Como puede ser analizado, el mantenimiento de la estructura o, como plantean Dessors y Moliner (1998), "la resistencia al cambio" es notable en el caso a estudiar. Para que un trabajador adopte un nuevo 
comportamiento, es necesario también que acepte renunciar al que ha adoptado hasta ese momento. Mejor aún, para que acepte el principio de un nuevo comportamiento, debe tener la posibilidad de criticar el comportamiento que era suyo hasta ese entonces.

La "resistencia al cambio" también es notable a la hora de tomar decisiones. En la cristalería, como en cualquier cooperativa, se pueden diferenciar claramente dos espacios de decisión: la Asamblea y el Consejo de Administración. El Consejo de Administración está conformado por once personas: el presidente, cinco representantes de la sección producción, tres de administración, uno de afiladura y depósito y uno de horno. Los cinco representantes de la sección producción son todos oficiales de banco.

Hay que tener en cuenta que en la conformación del Consejo no hay ninguna mujer. En la sección afiladura, la encargada es una mujer, ex-trabajadora de la empresa anterior, y la cual tuvo un papel sumamente importante a la hora de recuperar la empresa. Fue la encargada de recorrer casa por casa convenciendo a los oficiales de banco - piezas imprescindibles para recuperar la empresa - que volvieran a la fábrica.

Mirá, si está la gente, que son los artesanos, que es lo fundamental, sí se puede reabrir. Y entonces me preguntó si yo me podía ocupar de ver si estaba la gente, de empezar a recorrer. Yo le dije que sí, así que me puse en campaña y empecé a recorrerlos a todos (Entrevista: G., encargada sección afiladura).

Hoy sigue siendo la encargada de la sección. Es reconocida por sus compañeros, sin embargo no forma parte del Consejo de Administración.

Es notable como el predominio de la jerarquía del oficio en la organización interna de la empresa va a estructurar las relaciones de género internas. Al tratarse de un oficio altamente calificado y considerado de "varones" en el imaginario del colectivo de trabajo (integrado por varones y mujeres), esto va a llevar a un trato, por parte de los hombres, diferencial hacia la mujer, relegándola a los ámbitos de menor calificación y no otorgándole un lugar en el proceso decisorio. A la estructura de dominación masculina ${ }^{3}$ patriarcalista propia de la sociedad en general, se suma otra estructura de dominación masculina sostenida sobre la autoridad del oficio en el orden interno de la empresa, creando una doble inequidad en las relaciones sociales de género entre los miembros de un mismo colectivo de trabajo.

\section{Caso 2}

El segundo estudio de caso analizado se trata de una empresa metalmecánica dedicada a la construcción de carrocerías. En épocas de su normal funcionamiento, el colectivo de operarios se encontraba afiliado al gremio metalúrgico (UOM), gozando de puestos estables, con sueldos elevados y el $80 \%$ del personal empleado con calificación de oficiales de primera. El funcionamiento general de la empresa se sostenía sobre una lógica de funcionamiento industrial (EYMARDDUVERNAY, 1994).

Las mujeres con las que trabajamos - a quienes denominaremos A. y C. - son las esposas de dos trabajadores. Estas mujeres no estaban insertas en el mercado de trabajo. Esto permite pensar en contextos familiares relativamente estables, con relaciones primarias delimitadas fundamentalmente por el entorno familiar y barrial.

El conflicto, que tiempo después desencadenó en la recuperación de la planta, comenzó en 1997, y fueron las esposas de los trabajadores quienes estuvieron al frente de esa lucha.

Todos sabían que podían llegar a vaciar la empresa. Pero ninguno de los hombres se plantó enfrente para que no lo hicieran. Todos querían la continuidad. Había que hacer algo y nosotras lo hicimos. (Entrevista: A.)

A. y C. junto a otras cuatro mujeres, instalaron una carpa en una plaza frente a la planta que se sostuvo setenta y seis días. A los pocos días de su instalación, se incorporaron a la carpa unos veinte trabajadores despedidos, $\mathrm{y}$, lentamente, algunos organismos de derechos humanos, ONGs, agrupaciones estudiantiles universitarias sumaron su apoyo hasta constituirse en un contingente estable de 150 personas que ocupaban el predio donde se había asentado la carpa. En ese momento, la empresa negocia con los trabajadores y reincorpora a los despedidos.

Durante estos dos meses y medio, A. y C. no dejaron la carpa. Esta se constituyó en su "casa". Tengamos en cuenta que la mayor parte de sus vidas había estado dedicada a las tareas referidas a la reproducción familiar.

En febrero de 2000 la empresa declara la quiebra definitiva y cierra sus puertas. Es aquí donde comienza una segunda etapa de lucha ("de resistencia" la caracterizarán A. y C.). Nuevamente estas mujeres movilizan la instalación de una carpa, que esta vez durará nueve meses. Pero ninguna de las dos desarrollará su lucha desde ahí. A partir de este momento comienza para ambas un largo peregrinaje que dibuja dos recorridos: uno, que tiene como objetivo la visualización pública del conflicto; y el otro, que sigue los canales políticos-legales institucionales para obtener soluciones para su problema. Gracias a este peregrinaje es que consiguen legalmente la ocupación de la empresa y se conforman en cooperativa.

Del grupo de mujeres que originalmente organizan esta lucha en defensa de la fuente de trabajo de sus 
esposos, sólo A. y C. van a continuar los trámites legales correspondientes hasta obtener el permiso judicial para la apropiación temporaria de la empresa.

Las demás se fueron perdiendo. No sé, no les salía golpear y golpear puertas. Volantear en las esquinas. Nosotras nos olvidamos de nuestras casas, yo de ser abuela y ella dejaba a sus hijos. La calle nos endureció (Entrevista: C.).

Un dato importante es que si bien C. tenía sus hijos ya adultos, en el caso de A. sus hijos tenían seis y ocho año. Dice A, aludiendo a una división sexual del trabajo que no mostraba grandes cambios con respecto a la prevaleciente cuando ella era ama de casa solamente,

Mi marido ayudaba en la casa pero no era lo mismo. A veces yo llegaba y comía cualquier cosa porque no había nada preparado (Entrevista: A.).

Es interesante subrayar que A. y C. argumentan que todas las actividades que realizaron en esta lucha, las llevaron adelante presentándose como "esposas de obreros". Pocas veces daban sus nombres. Las cartas dirigidas a mostrar la problemática o a pedir soluciones en el ámbito público durante todo este proceso eran firmadas con la misma fórmula: “esposas de obreros", hipotecando la propia identidad tras la figura de los trabajadores. Sólo una vez dirimido el conflicto central y ocupando puestos dentro de la empresa ya recuperada comienzan a identificarse a sí mismas con sus nombres y apellidos.

Cuando los trabajadores se conforman en cooperativa de trabajo, ellas son elegidas en asamblea estatutaria como presidenta (C.) y vicepresidenta (A.) de la cooperativa de trabajo. Actualmente siguen desempeñando estos puestos dentro de la empresa, atendiendo proveedores, clientes, bancos y desarrollando las relaciones públicas de la empresa.

Cuando se les pregunta porque fueron ellas y no sus esposos quienes llevaron adelante esta lucha, ambas coinciden:

Los hombres estaban quebrados. Ellos eran los que tenían que mantener la casa. Ahí nosotras nos abríamos. Con todo lo que pasó, sufrieron mucho. Algunos se separaron, otros se emborrachaban. Los nuestros se quebraron. Ahí salimos nosotras. (Entrevista: C. y A.)

Retomando el concepto de género de Scott (1999), se debe mencionar que el conjunto de símbolos culturalmente significativos en el contexto de socialización de estas mujeres se asocia a los roles tradicionales de representaciones de "lo femenino y lo masculino" desde un modelo patriarcal de familia (esposas, amas de casa, encargadas de la reproducción y cuidado de sus hijos, sostenidas desde una pertenencia de clase trabajadora por sus esposos con sueldo relativamente altos y estabilidad del contrato laboral de tipo fordista). En segundo lugar, que el conjunto de normas que se generan a partir de aquel conjunto de símbolos y de sus interpretaciones, le asignaban a ellas, como mujeres, los valores de la comprensión, la aceptación, la resignación, la subalternidad como constitutivos de sus roles tradicionales de género. Y por último, las prácticas cotidianas sostenidas sobre aquellas representaciones y limitadas y habilitadas por el orden normativo, restringía sus prácticas al ámbito de lo privado, como madres y esposas “de", vedadas de la participación en el mercado de trabajo y carentes de protagonismo en el ámbito público de la política.

El cambio en sus prácticas cotidianas a partir de su participación en la lucha por la recuperación de la empresa donde trabajaban sus esposos, les permite una ruptura con estos estereotipos de género, un claro protagonismo en la lucha social y política que este conflicto involucraba, y desde la esfera económica, la obtención de un lugar como trabajadoras.

Sin embargo y a pesar de esta "lógica de la multiplicidad" (FERNÁNDEZ; BORAKIEVIVH, 2007) que tiene como escenario la lucha en el campo de las empresas recuperadas, desde su constitución y gestión cotidiana, basada en una organización autogestiva del trabajo y de la toma de decisiones, la "lógica de la delegación" sigue despuntando en ciertas prácticas y representaciones que estas mujeres encarnan, mostrando todo el peso de la "performatividad de género" (BUTLER, 2004) en relación a la identidad laboral que les estaba asignada:

A.: Mi marido una sola vez me acompañó a una marcha, con el esposo de C. y me dijo 'no te puedo acompañar nunca más'. Yo iba con mi hijo más chiquito que tenía cuatro años en ese momento, el esposo de C., C. y mi marido. Cuando vieron que llegamos, nosotros terminamos adelante [...] Ellos quedaban muy atrás pobres. Todo el mundo nos venía a saludar y dijo "nunca más". Además imaginate, los bombos, los papelitos, los micrófonos, los periodistas. Y subimos al atril. Me dijo "te desconozco".

C.: ¡Me cambiaron las mujeres!

A.: Nunca más me acompañó.

C.: Le cambiaron las mujeres (Entrevista: C. y A.).

Evidentemente la identidad de género de A. y C. ha sufrido modificaciones importantes, como así también la estructura interna de sus familias.

Si se reflexiona acerca de las significaciones que estas mujeres atribuyen a las prácticas y tareas que desarrollaron luego de la formación de la cooperati- 
va de trabajo, situando la atención en las "continuidades" o "discontinuidades" con respecto a sus trayectorias de vida, evidentemente aparecen dos planos superpuestos. En cuanto a la identidad que "los otros" (opinión pública, medios de comunicación, actores políticos, etc.) le otorgan - identidad social virtual (GOFFMAN, 1998) - se hace evidente la ruptura con su historia de vida anterior. Son productoras de sentido y prácticas permanentes en relación al proceso de recuperación de empresas. Son las referentes y la cara visible de la empresa, las que portan la historia de lucha, las que extienden los lazos de asociatividad que forjaron dentro de la empresa hacia otras empresas en condiciones similares a la de ellas.

Sin embargo y paradójicamente, hasta hace poco tiempo su identidad social estructurada por los mecanismos de apropiación, giraba en torno a desvalorizaciones que se reactualizaban en los sentidos construidos en las luchas por la fuente de trabajo. Recordemos que se presentaban como "esposas de...", tomaban la lucha para cubrir los espacios de los que el otro (sus esposos) se retiraba ("ellos están quebrados"). Esto marca continuidades en el rol que cumplían antes de que se iniciara la recuperación de la empresa.

Recién en la nueva instancia de la gestión de la empresa, aparecen ciertas rupturas con los roles tradicionales. Justamente, a partir de sus trabajos de gestión en la cooperativa, cobran envergadura en el "espacio público", entablando negociaciones cotidianas con funcionarios públicos, medios de comunicación, representantes de empresas de servicios, bancos, entre otros actores significativos en el proceso de toma y recuperación de la empresa.

Aparece un quiebre que indicaría cambios en distintos ámbitos. En primer lugar, en relación a los símbolos significativos en la representación de lo femenino (ya no son amas de casa que luchan por sus maridos, son trabajadoras dentro de la empresa). En segundo lugar, respecto a las normas (están nombradas por el estatuto de la cooperativa como presidenta y vice, asignándoseles funciones como tales). Por último, en las prácticas cotidianas dentro de la familia (han terciarizado parte de las tareas reproductivas). Al mismo tiempo, fueron reconocidas como representantes de la empresa en el ámbito económico y político. Se puede pensar que su identidad de género comienza a sufrir modificaciones.

\section{Conclusiones}

Teniendo en cuenta la descripción realizada a lo largo del trabajo sobre las trayectorias de vida de las mujeres implicadas en los procesos de lucha, se puede decir que en las dos empresas analizadas se encuentran cuatro variables que, de acuerdo al desarrollo particular de éstas en cada caso, permiten entender los diferentes lugares ocupados por las mujeres entrevistadas.

En primer lugar, las diferencias se dan en las estrategias de lucha durante el proceso de recuperación. En el primer caso analizado, el impulso inicial no fue de los trabajadores sino de actores ajenos a la empresa (Movimiento Nacional de Empresas Recuperadas y el intendente de la localidad). El proceso comenzó luego de un periodo prolongado de interrupción de la actividad de la empresa. Durante el proceso de recuperación, las mujeres, extrabajadoras, tuvieron dos funciones específicas. Por un lado, realizaron la custodia matutina y limpieza de las instalaciones. Por otro lado, una de ellas fue la encargada de contactar a los ex-trabajadores para poder comenzar el proceso de recuperación y posteriormente volver a producir. Es decir, sus tareas responden a la delegación de funciones que "otros" realizan para ellas. Lógica propia del modelo de división de trabajo sexual, en el cual la toma de decisiones corresponde al actor masculino y las tareas relativas a la reproducción del orden privado (limpieza y entrevistas con los actores involucrados en busca de compromisos) al actor femenino. En el segundo caso analizado, el proceso de lucha comienza antes del cierre de la fábrica. Desde los inicios, las mujeres son quienes llevan adelante la lucha por la recuperación de las fuentes laborales de sus esposos. En un primer momento también ocupándose de tareas reproductivas en la organización de las funciones dentro de las carpas de resistencia, pero en poco tiempo trascienden esa esfera y ganan espacio público, convirtiéndose en las referentes sociales de dicho proceso, capacitándose para ello y adquiriendo nuevas herramientas que les permitieran llevar adelante la lucha.

En segundo lugar, existe una gran diferencia en cuanto a la estructura organizativa posterior a la recuperación de la empresa. En el primer caso estudiado, se mantiene la estructura organizativa de la empresa anterior. Es decir, se mantienen los horarios, los espacios, y particularmente los cargos. El cambio sí se dio en la libre circulación por las secciones de la empresa y en el traspaso del oficio mediante la creación de la "Escuela de Artesanos". En el segundo caso, al no poder mantener la actividad original de producción de unidades carrozadas y teniendo que dedicarse sólo a la reparación de algunas unidades, esto implicó la necesaria reestructuración de la forma de organización de gestión del trabajo de la vieja empresa.

En tercer lugar, derivado del punto anterior y en relación a las actividades específicas de cada empresa, se pueden mencionar dos lógicas particulares de funcionamiento: en un caso "artesanal" o "doméstica" y en el otro "industrial". En el caso de la cristalería se puede notar como el predominio de la 
jerarquía del oficio (oficio considerado de varones) va a conducir a un trato, por parte de los hombres, diferencial hacia la mujer, relegándola a los ámbitos de menor calificación. En el segundo caso estudiado, si bien la actividad metalmecánica es predominantemente masculina, no implica cotos tan cerrados ya que algunos mecanizados que requieren motricidad fina han sido realizados por mujeres. Por otro lado, teniendo en cuenta que la empresa no pudo mantener su actividad original (construcción de carrozados), debiendo dedicarse reparaciones menores, las tareas de gestión se tornaron imprescindibles para mantener la "sustentabilidad" demandada por la ley de expropiación temporaria. Esto permitió que las tareas concretas que ellas realizaban en la búsqueda de financiamiento, de nuevos proyectos de inversión, de comercialización, etc., fueran trascendentes, y les permitió un espacio privilegiado en la toma de decisiones. De esta forma, la estructura interna de poder que la empresa tenía se reconfigura ya que si bien las mujeres no participan directamente del proceso productivo son nombradas presidenta y vice de la asamblea, gerenciando la totalidad de la empresa.

Por último, las diferencias se ven, aunque se trate de dos cooperativas, en los espacios asignados para la toma de decisiones y los lugares en ellos ocupados por las mujeres. En el primer caso, si bien existen los dos órganos establecidos por la Ley Nacional de Cooperativas - Consejo de Administración y Asamblea -, es el Consejo quien toma las decisiones. Éste, está conformado mayoritariamente por oficiales de banco, en su totalidad se trata de "viejos trabajadores" y varones. Si bien a las mujeres se les otorgó un rol fundamental en el proceso de recuperación y vuelta a la producción y son reconocidas por todos como parte importante del proceso productivo, no tienen participación en el Consejo, ya que no fueron elegidas por sus compañeros en laAsamblea. En el segundo caso analizado, se puede ver como las mujeres que protagonizaron el proceso de lucha, fueron elegidas por sus compañeros, mediante votación en Asamblea, para ocupar los cargos de mayor responsabilidad dentro de la estructura organizativa de la cooperativa.

A lo largo de este trabajo se ha tratado de dar cuenta de los procesos de subjetivación e intersubjetivación que se desprenden de las historias de vida de este grupo de mujeres, que, sostenidos sobre una malla de sentimientos, ideas, en suma, "representaciones sociales", se construyen en procesos de larga duración que no sólo se estructuran sobre las esferas económica y social sino también cultural, como un todo complejo. Las condiciones históricas de existencia de estos sujetos (mujeres y varones) son la clave para entender las significaciones que ellos otorgan a los bienes culturales, materiales, sociales y simbólicos. El análisis de las diferencias y similitudes entre las empresas estudiadas en un contexto de riesgo permite interpretar los procesos de cambio o continuidad en las relaciones de género implícitas en ellas y en la construcción de identidades laborales y de género.

\section{Referencias}

ANGÉLICO, H.; BUNEL, I. Las relaciones sociales en empresas del vidrio, organización del trabajo, relaciones laborales e identidad profesional. Buenos Aires: CEILCONICET, Serie Documentos de Trabajo, n. 21, 1989.

ARFUCH, L. Identidades, sujetos y subjetividades. Buenos Aires: Prometeo libros, 2005.

BOURDIEU, P. La dominación masculina. Barcelona: Ed. Anagrama, 2000.

BUTLER, J. Reescenificación de lo universal: hegemonía y límites del formalismo. In: BUTLER, J; LACLAU, E.; ZIZEK, S. Contingencia, hegemonía y universalidad: Diálogos contemporáneos en la izquierda. Buenos Aires: FCE, 2004. p. 49-93.

CASTEL R. La metamorfosis de la cuestión social. Una crónica del salariado. Buenos Aires: Paidós, 1997.

DESSORS, D.; MOLINIER, P. La psicodinámica del trabajo. In: DESSORS, D.; GUIHO-BAILLY, M. (Comp.) Organización del trabajo y salud. De la psicopatología a la psicodinamia del trabajo. Buenos Aires: Ed. Lumen, 1998. p. 11-22.

DUBAR, C.; DEMAZIERE, D. Analyser les entretiens biographiques. Paris: Nathan, 1997.

DUBAR, C. El trabajo y las identidades profesiones y personales. Revista Latinoamericana de Estudios del Trabajo, Buenos Aires: ALAST, año 7, n. 13, 2001.

EYMARD-DUVERNAY, F. Economía de las convenciones. Buenos Aires: Asociación Trabajo y Sociedad, 1994. 
FAJN, G. (Coord.). Fábrica y empresas recuperadas. Protesta social, autogestión y rupturas en la subjetividad. Buenos Aires: Ediciones del IMFC, 2004.

FERNÁNDEZ, A. M.; BORAKIEVICH, S. Fábricas recuperadas. La anomalía utogestiva. In: PRIMER ENCUENTRO INTERNACIONAL DE DEBATE: La economía de los trabajadores: autogestión y distribución de la riqueza. Fac. de Filosofía y letras. Buenos Aires: UBA, 2007.

GIDDENS, A. Modernidad e identidad del yo. El yo y la sociedad en la época contemporánea. Barcelona: Península, 1997.

GOFFMAN, E. Estigma. La identidad deteriorada. Buenos Aires: Amorrortu, 1998.

HIRATA, H.; KEORGOAT, D. La división sexual del trabajo. Permanencia y cambios. Buenos Aires: Piette, 1995.

LACLAU, E. Identidad y hegemonía: el rol de la universalidad en la constitución de lógicas políticas. In: BUTLER, J; LACLAU, E.; ZIZEK, S. Contingencia, hegemonía y universalidad: Diálogos contemporáneos en la izquierda. Buenos Aires: FCE, 2004. p. 49-93.

SCOTT, J. El género: una categoría útil para el análisis histórico. In: NAVARRO, M. et al. Sexualidad, género y roles sexuales. Buenos Aires: FCE, 1999. p. 37-75.

TOURAINE, A. La organización profesional de la empresa. In: FRIEDMANN, G.; NAVILLE, P. (Comp.): Tratado de Sociología del Trabajo. México: FEC, 1971. p. 389-400.

\section{Notas}

1 El proyecto fue sometido al Comité de Ética en Investigaciones con Seres Humanos de la UNR, con visto favorable. Fue solicitado el consentimiento libre y esclarecido de los sujetos entrevistados, a partir de un término de consentimiento para esta publicación, debidamente firmado por el investigado e investigador. Se aseguró la confidencialidad y privacidad de sus declaraciones, garantizando la no utilización de la información en perjuicio de los que participaran de la investigación y se observaron las normas legales y éticas para la investigación que envuelve seres humanos.

2 Giddens(1997,p. 39) aclara: "El estilo de vida refiere también a la toma de decisiones y los cursos de acción sujetos a condiciones de constricción material; semejantes patrones de estilo de vida, en ocasiones, pueden implicar también el rechazo más o menos deliberado de las formas ampliamente difundidas de comportamiento y consumo." En este sentido, Giddens nos previene del error de identificar únicamente el concepto de estilo de vida con el consumo y los propósitos de los "grupos o clases más opulentas." Si bien podríamos acordar que las clases humildes son cercenadas de la posibilidad de elección de un estilo de vida en su sentido amplio, este concepto apela también a "los diferentes accesos a las formas de autoactualización y realización individual" que la división en clases (u otras líneas de la desigualdad: género, etnia, etc.) determinan desde los sesgos de producción de diferencia, exclusión y marginalización.

3 EnsulibroLadominación masculina (2000), Bourdieu define género como un "conjunto de referencias" que estructura concreta y simbólicamente la percepción y la organización de la vida social, es el principal instrumento que establece la distribución de poder, en el sentido de un control diferenciado sobre los recursos materiales y simbólicos o de acceso a los mismos. Así la función legitimadora del género actúa cotidianamente y de diversas maneras dentro y fuera del ámbito de la familia.

\section{María de los Ángeles Dicapua}

angelesdicapua@yahoo.com.ar

Licenciada en Comunicación Social.

Profesora Titular. Directora del Centro de

Investigaciones y Estudios del Trabajo (CIET).

Facultad de Ciencia Política y RRII, Universidad Nacional de Rosario

\section{Melina Perbellini}

melinaperbellini@gmail.com

Licenciada en Ciencia Política.

Auxiliar de Investigación del CIET, Facultad de Ciencia Política y RRII, Universidad Nacional de Entre Ríos (UNR)

Jefa de Trabajos Prácticos, Facultad de Trabajo Social, UNR

\section{Evangelina Tifni}

evatifni@argentina.com

Licenciada en Ciencia Política.

Auxiliar de Investigación del CIET

Facultad de Ciencia Política y RRII, UNR.

\section{Universidad Nacional de Entre Ríos (UNR)}

Riobamba 250 bis. Monoblock 1.

Rosario - Santa Fe - Argentina 